\title{
Methylglyoxal as a new biomarker in patients with septic shock: an observational clinical study
}

\author{
Thorsten Brenner ${ }^{1 *}$, Thomas Fleming ${ }^{2 \dagger}$, Florian Uhle ${ }^{1}$, Stephan Silaff ${ }^{1}$, Felix Schmitt ${ }^{1}$, Eduardo Salgado ${ }^{1}$, \\ Alexis Ulrich ${ }^{3}$, Stefan Zimmermann ${ }^{4}$, Thomas Bruckner ${ }^{5}$, Eike Martin ${ }^{1}$, Angelika Bierhaus $^{2}$, Peter P Nawroth $^{2}$, \\ Markus A Weigand ${ }^{1}$ and Stefan Hofer $^{1}$
}

\begin{abstract}
Introduction: The role of reactive carbonyl species, such as methylglyoxal (MG), has been overlooked within the context of the sepsis syndrome. The aims of this study were to assess the impact of MG formation in different inflammatory settings and to evaluate its use for early diagnosis as well as prognosis of the sepsis syndrome.

Methods: In total, 120 patients in three groups were enrolled in this observational clinical pilot study. The three groups included patients with septic shock $(n=60)$, postoperative controls $(n=30)$, and healthy volunteers $(n=30)$. Plasma samples from patients with septic shock were collected at sepsis onset and after 24 hours and 4, 7, 14, and 28 days. Plasma samples from postoperative controls were collected prior to surgery, immediately following the end of the surgical procedure as well as 24 hours later and from healthy volunteers once. Plasma levels of MG were determined by high-performance liquid chromatography. Additionally, plasma levels of procalcitonin, C-reactive protein, soluble CD14 subtype, and interleukin-6 were determined.

Results: Patients with septic shock showed significantly higher plasma levels of MG at all measured times, compared with postoperative controls. MG was found to identify patients with septic shock more effectively - area under the curve (AUC): 0.993 - than procalcitonin (AUC: 0.844), C-reactive protein (AUC: 0.791), soluble CD14 subtype (AUC: 0.832), and interleukin-6 (AUC: 0.898) as assessed by receiver operating characteristic (ROC) analysis. Moreover, plasma levels of MG in non-survivors were significantly higher than in survivors (sepsis onset: ${ }^{*} P=0.018$ for 90 -day survival; ${ }^{* *} P=0.008$ for 28-day survival). Plasma levels of MG proved to be an early predictor for survival in patients with septic shock (sepsis onset: ROC-AUC 0.710 for 28-day survival; ROC-AUC 0.686 for 90-day survival).
\end{abstract}

Conclusions: MG was identified as a marker for monitoring the onset, development, and remission of sepsis and was found to be more useful than routine diagnostic markers. Further studies are required to determine the extent of MG modification in sepsis and whether targeting this pathway could be therapeutically beneficial to the patient.

Trial registration: German Clinical Trials Register DRKS00000505. Registered 8 November 2010.

\section{Introduction}

Sepsis represents an ongoing challenge in intensive care units (ICUs) and remains the most common cause of mortality in this setting [1,2]. Increased oxidative stress, as a consequence of the systemic inflammatory response, has been suggested as a major causative factor for the development and progression of the disease. However,

\footnotetext{
* Correspondence: thorsten.brenner@med.uni-heidelberg.de

${ }^{\dagger}$ Equal contributors

Deceased

'Department of Anesthesiology, University of Heidelberg, 110, Im

Neuenheimer Feld, D-69120 Heidelberg, Germany

Full list of author information is available at the end of the article
}

there is little conclusive evidence that a targeted treatment with antioxidants in patients with sepsis could be beneficial $[3,4]$. This may suggest the involvement of alternative mediators of cellular stress in the pathophysiology of sepsis.

Reactive carbonyl species (RCS) have emerged as effective mediators of cellular dysfunction. RCS are a heterogeneous group of reactive low-molecular-weight carbonyls, which are able to interact with various biomolecules, such as proteins, deoxyribonucleic acid, or phospholipids, resulting in structural distortions and functional impairment [5]. The detrimental effects of RCS are comparable 
to those caused by reactive oxygen species (ROS), except that RCS are significantly more stable and can readily diffuse out of the cell and have effects far from the original site of their formation [5]. Furthermore, several of the most reactive RCS are derived from glucose metabolism (in particular, glycolysis) [5]. As ROS require three stages of metabolism before they are produced, RCS can be viewed as providing a more direct insult to the macromolecular integrity of the cell.

Methylglyoxal (MG) is a highly reactive RCS, produced endogenously from the spontaneous degradation of triosephosphates-glyceraldehyde-3-phosphate (GA3P) and dihydroxyacetone phosphate (DHAP) - during glycolysis. It is estimated that approximately $0.089 \%$ of triosephosphates are converted to MG and that the total body rate of formation for a healthy adult human is about $3 \mathrm{mmol}$ per day [6]. The formation of MG is closely linked to the rate of glycolysis within the cell. It would be expected that under physiological conditions, where there is either an increase in glycolytic flux or an increased dependence on glycolysis for energy, the rate of MG formation would be increased $[7,8]$. This has proven to be the case in patients with diabetes mellitus, in which complications such as nephropathy, neuropathy, and retinopathy can be linked to increases in cellular levels of glycated proteins, which then are referred to as advanced glycation end products (AGEs) [9].

A clinical feature of patients with sepsis is hyperglycemia $[10,11]$. However, the consequences of elevated blood glucose in sepsis, particularly with respect to production of MG and its potential role in the development and progression of the disease, have yet to be fully investigated. The aims of this study were to assess the impact of MG formation in different inflammatory settings and to evaluate its use for early diagnosis as well as prognosis of sepsis.

\section{Materials and methods}

\section{Selection of patients and study procedures}

The observational clinical pilot study was approved by the local ethics committee (Ethics Committee of the Medical Faculty of Heidelberg: Trial Code Number S1232009/German Clinical Trials Register: DRKS00000505) and was conducted in the surgical ICU of the University Hospital of Heidelberg, Germany. Study and control patients or their legal designees provided written informed consent. In total, 120 patients in three groups were consecutively enrolled into the study from August 2009 to July 2010. The three groups were the following: (1) 60 patients with septic shock, according to the criteria of the International Sepsis Definitions Conference [12] (referred to as the septic group, or S) (Table 1), due to documented or suspected infection according to the criteria of the International Sepsis Forum Consensus Conference on Definitions of Infection in the ICU (Additional file 1: Table S1)
Table 1 Characteristics of 60 patients in the septic group

\begin{tabular}{lc}
\hline Septic group $(\mathbf{n}=\mathbf{6 0})$ & \\
\hline Demographic data & \\
Age, years & $70(64-76)$ \\
Male sex & $46(76.7 \%)$ \\
ASA status: I; II; III; IV; V & $1(1.7 \%) ; 11(18.3 \%) ; 29(48.3 \%) ;$ \\
& $15(25.0 \%) ; 1(1.7 \%)$
\end{tabular}

Primary site of infection (double-naming feasible)

\begin{tabular}{|c|c|c|}
\hline Lung & \multicolumn{2}{|c|}{$12(20.0 \%)$} \\
\hline Gastrointestinal tract & \multicolumn{2}{|c|}{$32(53.3 \%)$} \\
\hline Genitourinary tract & \multicolumn{2}{|c|}{$6(10.0 \%)$} \\
\hline Others & \multicolumn{2}{|c|}{$18(30.0 \%)$} \\
\hline Unknown & \multicolumn{2}{|c|}{$4(6.7 \%)$} \\
\hline \multicolumn{3}{|l|}{ Infection type } \\
\hline Gram-positive isolates & \multicolumn{2}{|c|}{$16(26.7 \%)$} \\
\hline Gram-negative isolates & \multicolumn{2}{|c|}{$16(26.7 \%)$} \\
\hline Combination of both & \multicolumn{2}{|c|}{$18(30.0 \%)$} \\
\hline $\begin{array}{l}\text { Suspected infection without } \\
\text { any microbiological finding }\end{array}$ & \multicolumn{2}{|c|}{$10(16.7 \%)$} \\
\hline \multicolumn{3}{|l|}{ Septic organ failures } \\
\hline Septic shock & \multicolumn{2}{|c|}{$60(100.0 \%)$} \\
\hline Acute renal failure & \multicolumn{2}{|c|}{$35(58.3 \%)$} \\
\hline Acute respiratory distress syndrome & \multicolumn{2}{|c|}{$49(81.2 \%)$} \\
\hline Acute liver failure & \multicolumn{2}{|c|}{$15(25.0 \%)$} \\
\hline Disease severity scoring & S/TO & $\mathrm{S} / \mathrm{T} 1$ \\
\hline APACHE II score & $33(27-38)$ & $33(28-39)$ \\
\hline SAPS & $75(66-86)$ & $73(64-84)$ \\
\hline SOFA score & $14(11-15)$ & $14(12-15)$ \\
\hline Clinical data & $\mathrm{S} / \mathrm{TO}$ & $\mathrm{S} / \mathrm{T} 1$ \\
\hline Norepinephrine, $\mu \mathrm{g} / \mathrm{kg}$ per minute & $0.20(0.06-0.31)$ & $0.20(0.08-0.32)$ \\
\hline Maximum heart rate, $1 /$ minute & $115(97-130)$ & $113(102-127)$ \\
\hline Minimum MAP, mm Hg & $57(50-64)$ & $60(54-66)$ \\
\hline $\mathrm{FiO}_{2}$, none & $0.65(0.50-0.80)$ & $0.58(0.46-0.70)$ \\
\hline
\end{tabular}

Data are presented as number (percentage) or as median (with quartiles). APACHE II, Acute Physiology and Chronic Health Evaluation II; ASA status, physical status classification system according to the American Society of Anesthesiologists; $\mathrm{FiO}_{2}$, fraction of inspired oxygen; MAP, mean arterial pressure; SAPS, Simplified Acute Physiology Score; SOFA, Sequential Organ Failure Assessment.

[13]; (2) 30 postoperative controls following major abdominal surgery without any evidence of infection (the postoperative group, or P) (Table 2); and (3) 30 healthy volunteers (the volunteer group, or V) (Table 2). Blood samples from patients with septic shock were collected at sepsis onset (T0) and 24 hours (T1), 4 days (T2), 7 days (T3), 14 days (T4), and 28 days (T5) later. Blood samples from the postoperative group were collected prior to surgery (T0), immediately following the end of the surgical procedure (T1), and 24 hours later (T2). Blood samples from the volunteer group were collected once (T0). 
Table 2 Characteristics of $\mathbf{3 0}$ patients in the postoperative group and $\mathbf{3 0}$ individuals in the volunteer group

Postoperative group $(n=30)$

Demographic data

Age, years

$62(57-70)$

Male sex

$16(53.3 \%)$

ASA status: I; II; III; IV; V

$0(0.0 \%) ; 9$ (30.0\%); 20 (66.7\%); $1(3.3 \%) ; 0(0.0 \%)$

Site of surgery (double-naming feasible)

Liver

$7(23.3 \%)$

Pancreas

$11(36.7 \%)$

Gastrointestinal

$27(90.0 \%)$

Volunteer group $(n=30)$

Demographic data

Age, years

$26(24-28)$

Male sex

$19(63.3 \%)$

ASA status: I; II; III; IV; V

21 (70.0\%); 9 (30.0\%); 0 (0.0\%); 0 (0.0\%); $0(0.0 \%)$

Data are presented as number (percentage) or as median (with quartiles). ASA status, physical status classification system according to the American Society of Anesthesiologists.

\section{Immunoassays}

Plasma concentrations of total antioxidant capacity (TAC), methylglyoxal-derived advanced glycation end products (MG-AGE), and interleukin-6 (IL-6) were measured by using enzyme-linked immunosorbent assay (ELISA) kits in accordance with the instructions of the manufacturer (TAC and MG-AGE: Biocat, Heidelberg, Germany; IL-6: R\&D Systems, Minneapolis, MN, USA). Plasma levels of soluble CD14 subtype (sCD14-ST) were measured by using the Pathfast Presepsin chemiluminescent enzyme immunoassay in accordance with the instructions of the manufacturer (Mitsubishi Chemical, Tokyo, Japan).

\section{Methylglyoxal measurements with high-performance liquid chromatography}

The concentrations of MG in plasma were determined by derivatization with 1,2-diamino-4,5-dimethoxybenzene and high-performance liquid chromatography of the quinoxaline adduct by fluorescence detection $[14,15]$.

\section{Preparation of peripheral blood mononuclear cells}

Peripheral blood mononuclear cells (PBMCs) were separated immediately after blood collection. Ethylenediaminetetraacetic acid (EDTA)-anticoagulated whole blood was loaded carefully onto a lymphocyte separation medium (PAA Laboratories GmbH, Pasching, Austria) and centrifuged for 25 minutes at 1,200 rpm without brakes at $4^{\circ} \mathrm{C}$. The PBMC-containing band was aspirated, and the cells were washed three times with $\mathrm{NaCl} 0.9 \%$.
RNA extraction and quantitative polymerase chain reaction RNA extraction was performed by using the columnbased RNeasy Plus Mini Kit (Qiagen, Hilden, Germany) in accordance with the instructions of the manufacturer. RNA (250 ng) was reverse-transcribed by using the QuantiTect Reverse Transcription Kit (Qiagen) in accordance with the instructions of the manufacturer. Subsequent real-time polymerase chain reaction (PCR) analysis was performed on a StepOnePlus PCR cycler (Applied Biosystems, Weiterstadt, Germany) by using predesigned TaqMan assays for glyoxalase-1 (GLO-1) (assay ID Hs00198702_m1) and $\beta$-Actin (assay ID Hs99999903_m1). PCRs were set up by using the TaqMan Universal PCR Master Mix (Applied Biosystems, Weiterstadt, Germany). All experiments were run in triplicate. Results are interpreted by calculating the change in cycle threshold $(\Delta \mathrm{Ct})$ value $(\mathrm{Ct} \beta$-actin $-\mathrm{Ct}$ GLO-1) of each sample.

\section{Statistical analysis}

The present clinical investigation was conducted as a pilot study. Group sizes were set at 60 individuals in the septic group and 30 individuals in both the healthy and postoperative groups. Resulting study data was entered into an electronic database (Microsoft Excel 2010, Microsoft Corporation, Redmond, WA, USA) and evaluated by using SPSS software (Statistical Product and Services Solutions, Version 20.0, SPSS Inc., Chicago, IL, USA) or Graphpad Prism for Macintosh (Version 5.0f, GraphPad Software, San Diego, CA, USA). Categorical data were summarized by means of absolute and relative frequencies. Quantitative data were summarized by using medians (with quartiles). Wherever appropriate, data was visualized by using line or bar charts. The Kolmogorov-Smirnov test was applied to check for normal distribution. Owing to non-normally distributed data in this study, non-parametric methods for evaluation were used. Furthermore, a receiver operating characteristic (ROC) curve was calculated with suitable parameters in order to create cutoff values to determine the diagnostic or prognostic value of each parameter with regard to the diagnosis as well as the prognosis of sepsis. Comparisons of the areas under two or more correlated ROC curves were performed as described by Delong et al. [16]. Correlation analysis was performed calculating Spearman-Rho (r). A $P$ value of less than 0.05 was considered statistically significant. The following symbols were used with regard to higher orders of significance: ${ }^{*} P<0.05,{ }^{* *} P<0.01$, ${ }^{* * *} P<0.001$.

\section{Results}

In total, 120 patients in three groups were subjected for evaluation. A detailed characterization of the different groups is presented in Tables 1 and 2 . 


\section{Kinetics of methylglyoxal-derived carbonyl stress in human sepsis}

In patients with septic shock $(n=60)$, plasma concentrations of MG were significantly increased in comparison with healthy volunteers $(n=30)$ (Figure 1a) and compared with postoperative controls $(n=30)$ (Figure $1 b)$. Similar observations could be made for other routine infection and inflammation markers such as C-reactive protein (CRP), procalcitonin (PCT), IL-6, and sCD14-ST (Table 3 and Additional file 2: Table S2). However, MG proved to be superior for the identification of patients with septic shock (S/T0 versus P/T2) as assessed by area under the curve (AUC) comparisons of the related ROC curves (Figure 2 and Table 4). Besides, plasma levels of MG were shown to be independent of the septic focus (lung, gastrointestinal tract, genitourinary tract, and so
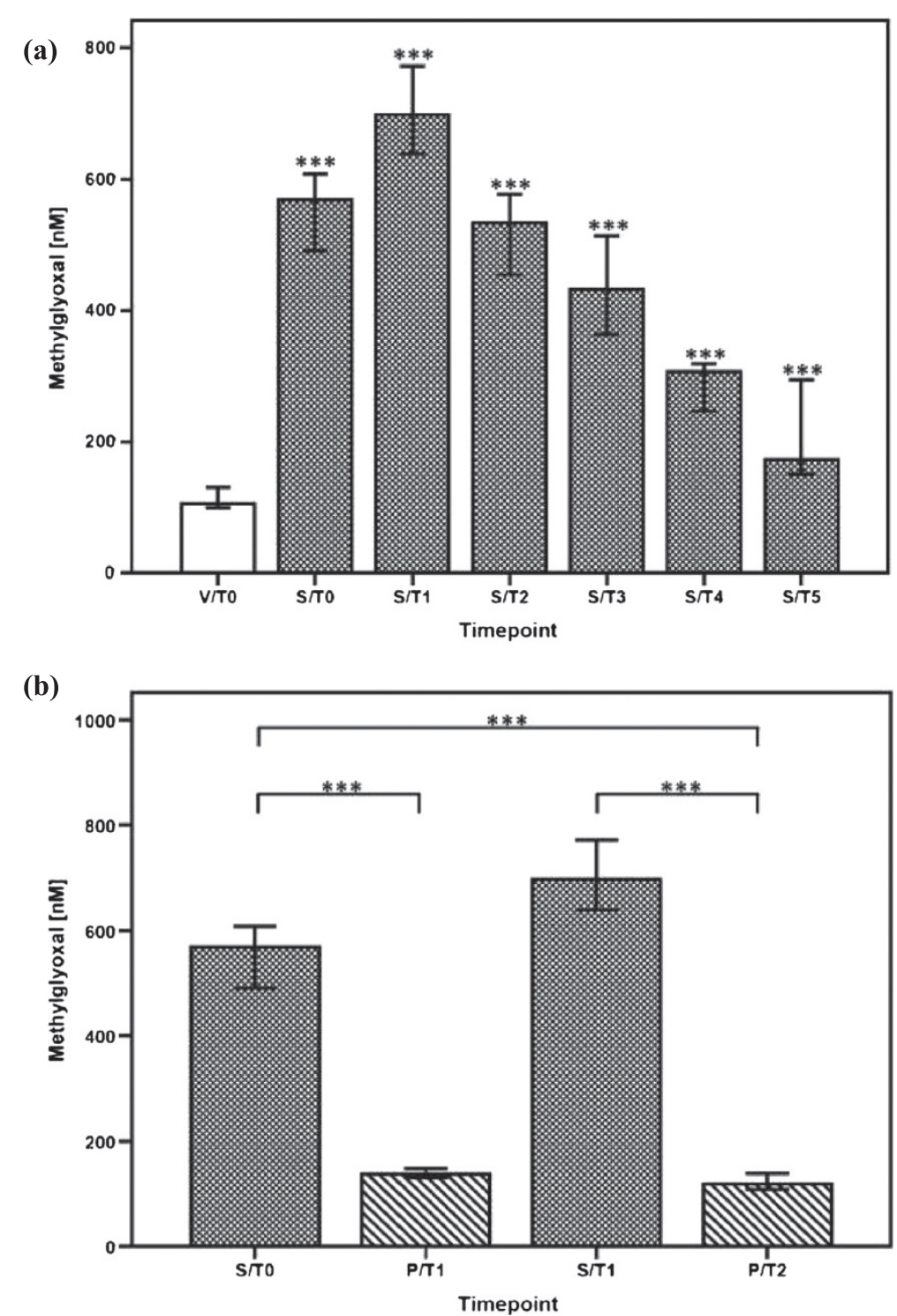

Figure 1 Plasma levels of methylglyoxal in different inflammatory settings in humans. (a) Comparison of plasma methylglyoxal measurements in healthy volunteers $(n=30$, white bar) and patients with septic shock $(n=60$, white-spotted bars in black color). Plasma levels of methylglyoxal in patients with septic shock are presented at sepsis onset (S/T0) and 24 hours (S/T1), 4 days (S/T2), 7 days (S/T3), 14 days (S/T4), and 28 days (S/T5). Plasma methylglyoxal measurements in healthy volunteers were performed once ( $/ / T 0)$. Data in bar charts are presented as medians and $95 \%$ confidence intervals (Cls). With regard to symbolism and higher orders of significance: ${ }^{* *} P<0.001$. Asterisks refer to pairwise comparisons between healthy volunteers and patients with septic shock at the presented time points. (b) Comparisons of plasma methylglyoxal measurements in postoperative controls following major abdominal surgery $(n=30$, white-striped bars in black color) and patients with septic shock ( $n=60$, white-spotted bars in black color). Plasma levels of methylglyoxal in patients with septic shock are presented at sepsis onset (S/T0) and 24 hours (S/T1) later. Plasma methylglyoxal measurements in postoperative controls are presented immediately after the end of the surgical procedure (P/T1) and 24 hours afterwards (P/T2). Data in bar charts are presented as medians and $95 \%$ Cls. With regard to symbolism and higher orders of significance: ${ }^{* * *} P<0.001$. 
Table 3 Plasma levels of C-reactive protein, procalcitonin, interleukin-6, soluble CD14 subtype, blood glucose, and total antioxidant capacity in the volunteer (V/TO), postoperative (P/TO, P/T1, P/T2), and septic (S/T0, S/T1) groups

\begin{tabular}{|c|c|c|c|c|c|c|c|c|c|c|c|}
\hline \multirow[t]{2}{*}{ Parameters } & \multirow{2}{*}{$\begin{array}{l}\text { Volunteer group }(n=30) \\
\text { V/T0 }\end{array}$} & \multicolumn{6}{|c|}{ Postoperative group $(n=30)$} & \multicolumn{4}{|c|}{ Septic group $(n=60)$} \\
\hline & & $\mathrm{P} / \mathrm{TO}$ & & $\mathrm{P} / \mathrm{T} 1$ & & $\mathrm{P} / \mathrm{T} 2$ & & $\mathrm{~S} / \mathrm{TO}$ & & $\mathrm{S} / \mathrm{T} 1$ & \\
\hline CRP, mg/L & 2.0 & 4.3 & $* * *$ & 4.3 & $* * *$ & 110.2 & $* * *$ & 169.2 & $* * *$ & 190.4 & *** \\
\hline$P C T, \mu g / L$ & 0.06 & 0.07 & ns & 0.10 & $* *$ & 0.82 & $* * *$ & 4.63 & $* * *$ & 7.17 & *** \\
\hline IL-6, pg/mL & 0.0 & 0.0 & $* *$ & 215.3 & $* * *$ & 132.5 & $* * *$ & 500.5 & $* * *$ & 337.6 & $* * *$ \\
\hline sCD14-ST, pg/mL & 123.0 & 208.5 & $* * *$ & 711.0 & $* * *$ & 680.0 & $* * *$ & 1817.5 & $* * *$ & 2309.0 & $* * *$ \\
\hline Blood glucose, mg/dL & 85.0 & 102.5 & $* *$ & 140.0 & $* * *$ & 116.0 & $* * *$ & 128.5 & $* * *$ & 139.0 & $* * *$ \\
\hline $\mathrm{TAC}, \mathrm{mM}$ & 0.02 & 0.06 & $* * *$ & 0.10 & $* * *$ & 0.12 & $* * *$ & 0.15 & $* * *$ & 0.13 & $* * *$ \\
\hline
\end{tabular}

Data are presented as medians. Results of pairwise comparisons refer to intergroup comparisons of each presented time point in the postoperative (P/T0, $\mathrm{P} / \mathrm{T} 1, \mathrm{P} / \mathrm{T} 2)$ and septic (S/TO, S/T1) groups with the volunteer group (V/T0). With regard to symbolism and higher orders of significance: ${ }^{*} P<0.05 ;{ }^{* *} P<0.01$; ${ }^{* *} P<0.001 ;$ ns, not statistically significant. The central lab of the University Hospital of Heidelberg provides precise values for C-reactive protein (CRP) beginning from 2 mg/L. Lower CRP values are simply denoted less than $2.0 \mathrm{mg} / \mathrm{L}$. For statistical analyses, all values less than $2.0 \mathrm{mg} / \mathrm{L}$ were put on a level of $2.0 \mathrm{mg} / \mathrm{L}$. Precise values for PCT are available beginning from $0.05 \mu \mathrm{g} / \mathrm{L}$. Lower PCT values are simply denoted less than $0.05 \mu \mathrm{g} / \mathrm{L}$. For statistical analyses, all values less than $0.05 \mu \mathrm{g} / \mathrm{L}$ were put on a level of $0.05 \mu \mathrm{g} / \mathrm{L}$. IL-6, interleukin-6; PCT, procalcitonin; sCD14-ST, soluble CD14 subtype; TAC, total antioxidant capacity.

on) as well as the underlying pathogen (Gram-positive isolates, Gram-negative isolates, both, and suspected infection without any microbiological finding) (data not shown).

\section{Methylglyoxal-derived carbonyl stress in human sepsis -} Relevance for the progression of the disease?

Within a 28-day and 90-day observation period, plasma levels of MG at sepsis onset were significantly higher in non-survivors than in the corresponding survivors (Additional file 3: Figure S1a and Additional file 4: Figure S2a). Accordingly, plasma levels of MG proved to be an early predictor for survival in patients with septic shock (sepsis onset: ROC-AUC 0.710; cutoff: $591.8 \mathrm{nM} \rightarrow$ sensitivity: 0.700 ; 1 -specificity: 0.300 for 28-day survival; ROC-AUC 0.686; cutoff: $568.4 \mathrm{nM} \rightarrow$ sensitivity: 0.655 ; 1 -specificity: 0.308 for 90-day survival) (Additional file 3: Figure S1b and Additional file 4: Figure S2b). In contrast, plasma levels of MG did not differ significantly between patients with sepsis-associated organ failures-for example, acute liver failure, acute renal failure, and acute respiratory distress syndrome-in comparison with those septic patients with unimpaired organ function (data not shown). One main effect of MG is the post-translational modification of proteins to form AGEs. Accordingly, the levels of plasma MG-AGEs reached their

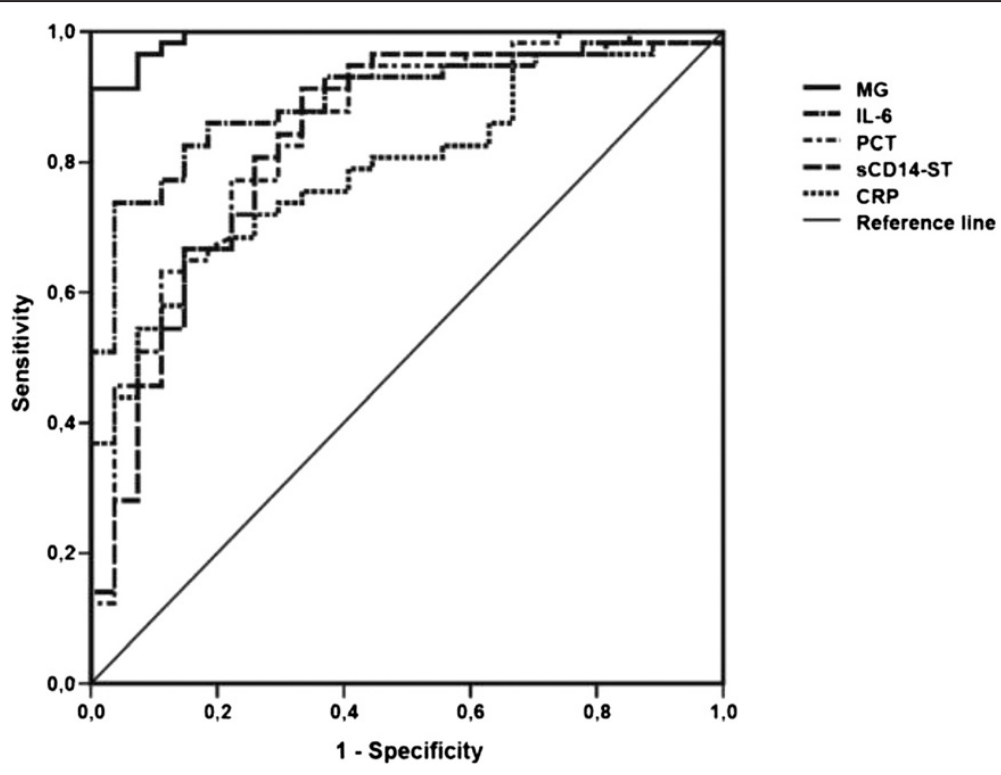

Figure 2 Diagnostic value of methylglyoxal (MG), C-reactive protein (CRP), procalcitonin (PCT), interleukin-6 (IL-6), and soluble CD14 subtype (sCD14-ST) for identification of patients with sepsis. Receiver operating characteristic curves for plasma levels of MG (continuous line), IL-6 (tight alternating dashed line), CRP (short dashed line), PCT (wide alternating dashed line), and SCD14-ST (long dashed line) in patients with septic shock at sepsis onset $(S / T 0, n=60)$ and postoperative controls 24 hours after major abdominal surgery $(P / T 2, n=30)$. 
Table 4 Diagnostic value of methylglyoxal, C-reactive protein, procalcitonin, interleukin-6, and soluble CD14 subtype for identification of patients with sepsis

\begin{tabular}{|c|c|c|c|c|c|}
\hline Parameter & AUC (S/T0 vs. P/T2) & Cutoff & Sensitivity & 1-Specificity & Results of pairwise comparisons \\
\hline$\overline{M G}, \mathrm{nM}$ & 0.993 & 281.1 & 0.912 & 0.000 & \\
\hline$C R P, m g / L$ & 0.791 & 152.8 & 0.667 & 0.148 & $<0.001^{* * *}$ \\
\hline$P C T, \mu g / L$ & 0.844 & 1.46 & 0.772 & 0.222 & $0.002^{* *}$ \\
\hline IL-6, pg/mL & 0.898 & 401.0 & 0.737 & 0.037 & $0.007^{* *}$ \\
\hline sCD14-ST, pg/mL & 0.832 & 825 & 0.912 & 0.333 & $<0.001^{* * *}$ \\
\hline
\end{tabular}

Receiver operating characteristic (ROC) analysis was performed with suitable parameters in order to create cutoff values to determine the diagnostic value of each parameter with regard to the diagnosis of sepsis (S/T0 versus P/T2). Area under the curve (AUC) comparisons of the related ROC curves refer to pairwise comparisons of each parameter with MG. With regard to symbolism and higher orders of significance: ${ }^{* *} P<0.01 ;{ }^{* * *} P<0.001$. CRP, C-reactive protein; IL-6, interleukin-6; MG, methylglyoxal; ns, not statistically significant; PCT, procalcitonin; sCD14-ST, soluble CD14 subtype.

maximum at 24 hours after sepsis onset in patients who developed septic shock. However, plasma levels of MGAGEs failed to be significantly higher in patients with septic shock compared with healthy volunteers. In contrast, plasma levels of MG-AGEs were significantly elevated in patients with septic shock in comparison with postoperative controls at 24 hours (Figure $3 \mathrm{a}$ and $3 \mathrm{~b}$ ).

\section{Methylglyoxal-derived carbonyl stress in human sepsis - a consequence of increased methylglyoxal formation?}

Blood sugar levels and the corresponding rate of glycolysis were hypothesized to have a relevant influence on plasmatic MG levels. Accordingly, patients with septic shock and postoperative controls showed significantly increased blood sugar levels over the entire study period in comparison with healthy volunteers (Table 3). However, there was only a weak correlation between plasma levels of MG and blood sugar levels $(r=0.139)$ in all study groups, implicating the contribution of other factors (for example, oxidative stress) to the measured increased plasma levels of MG than solely hyperglycemia. Healthy volunteers were shown to experience a low grade of oxidative stress, as indirectly assessed by plasma TAC. In contrast, postoperative controls already showed increased levels of oxidative stress prior to surgery (P/T0), which further increased over time after the surgical procedure (P/T1, P/T2). Compared with both control groups, patients with septic shock were found to have the highest level of oxidative stress within 24 hours after sepsis onset (S/T0, S/T1) (Table 3) and plasma TAC was found to positively correlate with MG plasma levels $(r=0.348)$ in all study groups.

Methylglyoxal-derived carbonyl stress in human sepsis a consequence of inhibited methylglyoxal detoxification? In comparison with healthy volunteers, expression of GLO-1, the key enzyme of the MG detoxification pathway, was shown to be significantly reduced in patients with septic shock within the first 24 hours after sepsis onset (S/T0, S/T1) (Figure 4).

\section{Discussion}

In this study, MG was identified as a marker for monitoring the onset, development, and remission of sepsis. It was found to be more useful than routine diagnostic markers, such as CRP, PCT, IL-6, and sCD14-ST. This finding is of great interest, as it has been suggested that the currently used biomarkers for sepsis do not have either the specificity or sensitivity to be routinely used in clinical practice [17].

The major source of endogenous MG is from the nonenzymatic degradation of the triosephosphate intermediates within glycolysis. Under conditions of increased glycolytic flux, such as hyperglycemia (a symptom often observed in patients with sepsis), it would be safe to assume that there should be an increase in MG production. In this study, blood sugar levels were significantly elevated in patients with septic shock. Measured plasma MG in these patients was also elevated, but only a weak correlation with blood glucose levels could be shown. This would suggest that there are other factors contributing to the measured increased plasma levels of MG than only hyperglycemia.

In this study, patients with septic shock characteristically have an increased state of oxidative stress and this correlates positively with plasma MG levels, suggesting that a relationship exists between these two forms of metabolic stress. GLO-1, the major pathway for detoxification of MG, is a glutathione (GSH)-dependent enzyme [18]. Previous studies have shown that under conditions of oxidative stress, both GSH and nicotinamide-adeninedinucleotide-phosphate-hydrate (NADPH) are depleted, which in turn decreases the in situ activity of GLO-1 [19], thereby increasing the concentration of MG. The loss of essential cofactors in a situation of increased oxidative stress could therefore be one explanation for the increased plasma MG observed in the patients with sepsis. However, it was also found that patients with sepsis syndrome had a significant reduction in the expression of mononuclear GLO-1, suggesting that patients with sepsis are more susceptible to the accumulation of MG through the loss of the GLO-1 rather than the loss of an 
(a)

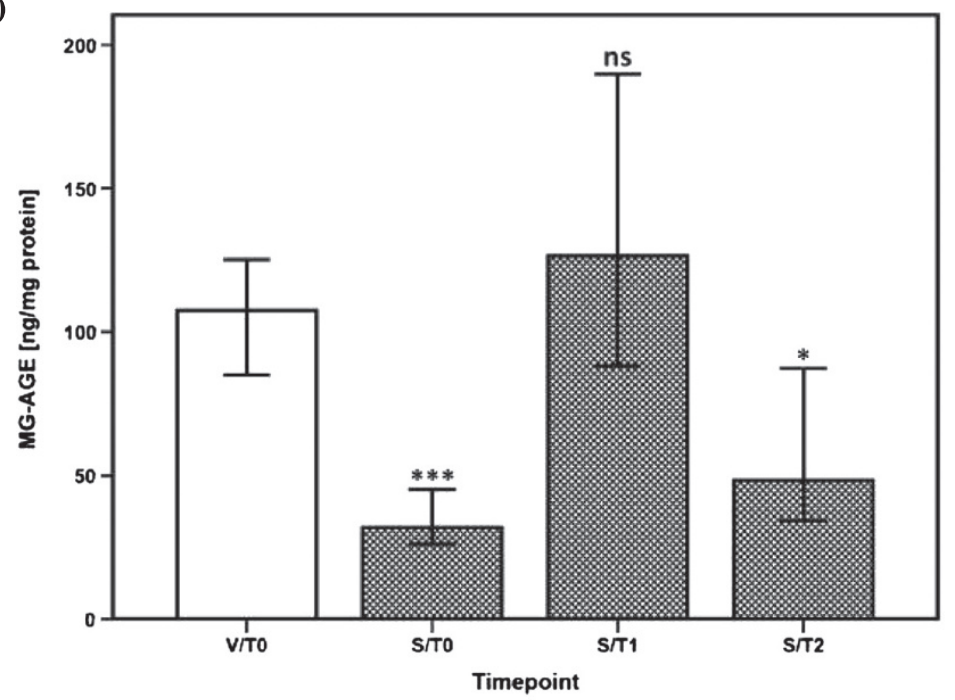

(b)

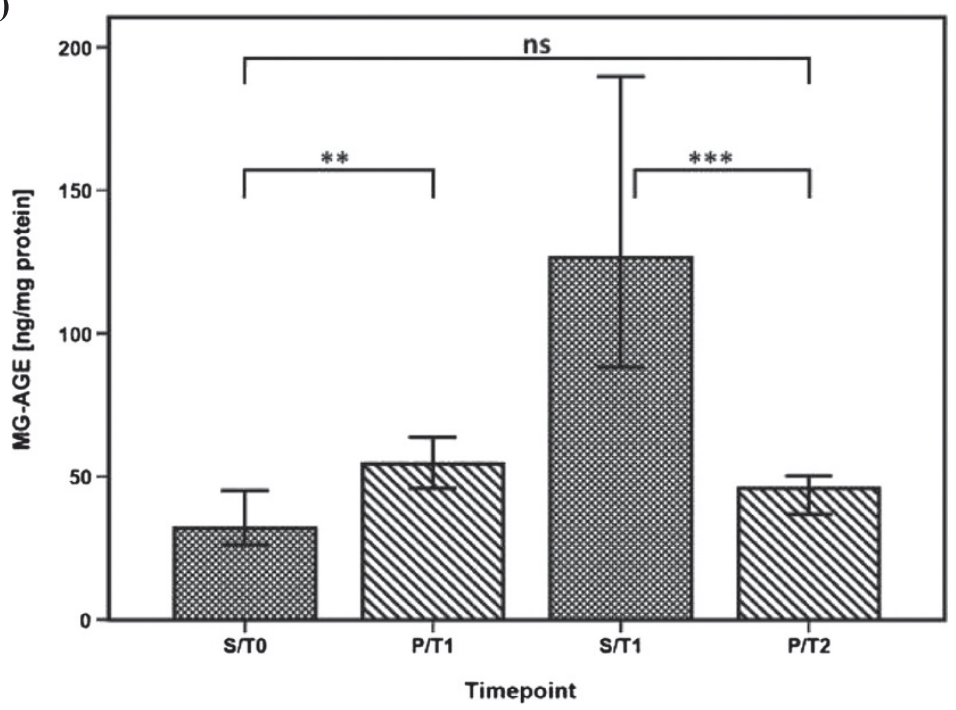

Figure 3 Methylglyoxal-derived advanced glycation end product (MG-AGE) formation in different inflammatory settings in humans. (a) Comparisons of plasmatic MG-AGE formation in healthy volunteers $(n=30$, white bar) and patients with septic shock $(n=60$, white-spotted bars in black color). Plasma levels of MG-AGEs in patients with septic shock are presented at sepsis onset (S/T0) and 24 hours (S/T1) and 4 days (S/T2) later. Plasma measurements of MG-AGEs in healthy volunteers were performed once (V/T0). Data in bar charts are presented as medians and $95 \%$ confidence intervals (Cls). With regard to symbolism and higher orders of significance: ${ }^{*} P<0.05$; ${ }^{* *} P<0.001$. Asterisks refer to pairwise comparisons between healthy volunteers and patients with septic shock at the presented time points. (b) Comparisons of plasma MG-AGE formation in postoperative controls following major abdominal surgery $(n=30$, white-striped bars in black color) and patients with septic shock $(n=60$, white-spotted bars in black color). Plasma levels of MG-AGEs in patients with septic shock are presented at sepsis onset (S/T0) and 24 hours (S/T1) later. Plasma measurements of MG-AGEs in postoperative controls are presented immediately after the end of the surgical procedure (P/T1) and 24 hours afterwards (P/T2). Data in bar charts are presented as medians and 95\% Cls. With regard to symbolism and higher orders of significance: ${ }^{* *} P<0.01 ;{ }^{* * *} P<0.001$. ns, not statistically significant.

essential cofactor. It has been proposed that the engagement of the receptor for advanced glycation end products (RAGE) by inflammatory mediators, such as carboxylmethyl-lysine (CML) and high-mobility group box protein-1 (HMGB1), can reduce expression of GLO-1 [20]. In this study, the cell-surface expression of RAGE was increased in the monocytes of patients with septic shock; however, there was also a significant reduction in the plasma concentration of the classic RAGE ligands (data not shown). It has also been proposed that hyperglycemic conditions, such as those observed in diabetes, can directly reduce GLO-1 activity. However, the underlying mechanism for this effect remains unclear [21]. 


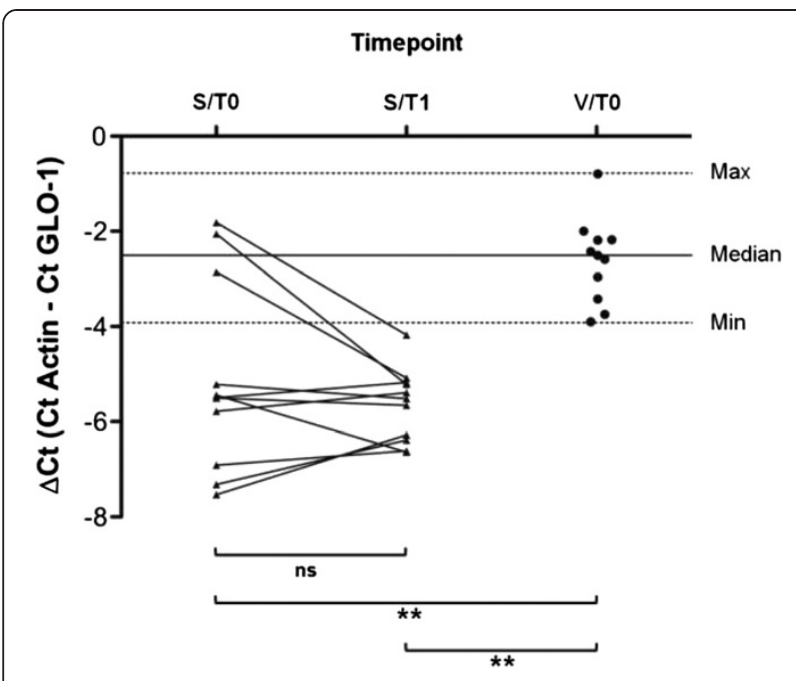

Figure 4 Methylglyoxal-detoxifying capacity in different inflammatory settings in humans. Glyoxalase-1 (GLO-1) expression in peripheral blood mononuclear cells of patients with septic shock compared with healthy volunteers is shown. Samples from 11 patients with septic shock -at onset (S/T0) and 24 hours (S/T1) later-and 11 healthy volunteers (V/TO) were analyzed by quantitative reverse transcription-polymerase chain reaction. Dotted horizontal lines represent the minimum and maximum change in cycle threshold $(\Delta \mathrm{Ct})$ values of healthy volunteers, and solid lines shows the median accordingly. With regard to symbolism and higher orders of significance: ${ }^{*} P<0.01$. ns, not statistically significant.

One of the primary effects of elevated MG is the posttranslational modification of proteins to form AGEs $[22,23]$. This study could show that the concentration of MG-AGEs in plasma paralleled the levels of MG and that the highest concentrations were observed 24 hours after the onset of sepsis. This may simply reflect a cause-and-effect relationship in the plasma; however, it may also indicate an increased turnover of MG-modified or damaged proteins within the tissue [6]. Such modified proteins are degraded by cellular proteolysis, releasing not only modified peptides but also the modified amino acid, which is eventually excreted in the urine [6]. Several studies have shown that MG-derived hydroimidazolone (MG-H1) [24], the adduct formed from MG modification of arginine, is the major quantitative modified adduct excreted in humans and rats $[25,26]$. Increased excretion of this modified product, particularly in diabetes mellitus, is associated with the development and progression of complications, such as neuropathy, nephropathy, and retinopathy [9,27-30]. It has also been shown that MG-AGEs, specifically MG-H1, can interact with RAGE. Monocytes can bind, internalize, and degrade albumin which has been minimally modified by MG, leading to synthesis and secretion of pro-inflammatory mediators such as IL-1 $\beta$, macrophage colony-stimulating factor, and tumor necrosis factor-alpha. It has been suggested that this binding is mediated by RAGE [31-35], making it possible that the high circulating levels of MG-AGE proteins found in patients with sepsis could activate circulating monocytes by ligating RAGE. This may result in the downregulation of GLO-1 and the induction of the pro-inflammatory phenotype observed in patients with septic shock. Further in vitro studies using MGmodified proteins, modified to a similar extent as those observed in patients with sepsis, are required to confirm this observation.

The findings of increased MG and MG protein damage in sepsis suggest that MG metabolism is an important and generally overlooked biochemical pathway for the induction of cellular dysfunction or inflammation (or both) in sepsis. Furthermore, prevention of MG overload might represent a new therapeutic option in sepsis. Unfortunately, there are currently no MG scavengers approved for clinical use. As in diabetes, tight blood glucose control in patients with sepsis may offer a strategy for reducing the amount of MG accumulation. Accordingly, recent clinical guidelines for the treatment of sepsis have recommended that blood glucose levels should be maintained at not more than $180 \mathrm{mg} / \mathrm{dL}$ [36]. An alternative treatment strategy could be to increase GLO-1 activity. It has been shown in vitro that GLO-1 transcription is regulated by the antioxidant transcription factor nuclear factor (erythroid-derived 2)-like 2 (Nrf2) [37]. Activation of this transcription factor by isothiocyanates, such as sulphoraphane, can lead to increased GLO-1 activity and decreased MG and MGderived AGEs [38]. Such compounds are found in high abundance in cruciferous vegetables. However, the effect of such treatment in vivo remains unknown and requires further investigation to determine whether it would be effective in acute illnesses such as sepsis.

Further investigations are required to determine whether the diagnostic and prognostic value of MG can be confirmed and validated in a large study cohort. Moreover, to link the clinical observations of our study to functional implications, it needs to be evaluated in vitro, whether MG modifications are responsible for the cellular dysfunction or inflammation (or both) observed in sepsis. Critical to this process would be to identify the cellular targets involved in this modification. It could already be shown that mitochondrial proteins are particularly susceptible to modification by MG [39]. The ELISA-based method used to detect MG-AGEs in this study is unable to differentiate whether the MG-AGEs measured are complete proteins, peptides, or the free amino acids. In future studies, the measurement of both MG and MG-AGEs should be performed by using the gold-standard technique of stable isotope dilution, liquid chromatography tandem mass spectroscopy, which could provide robust identification, 


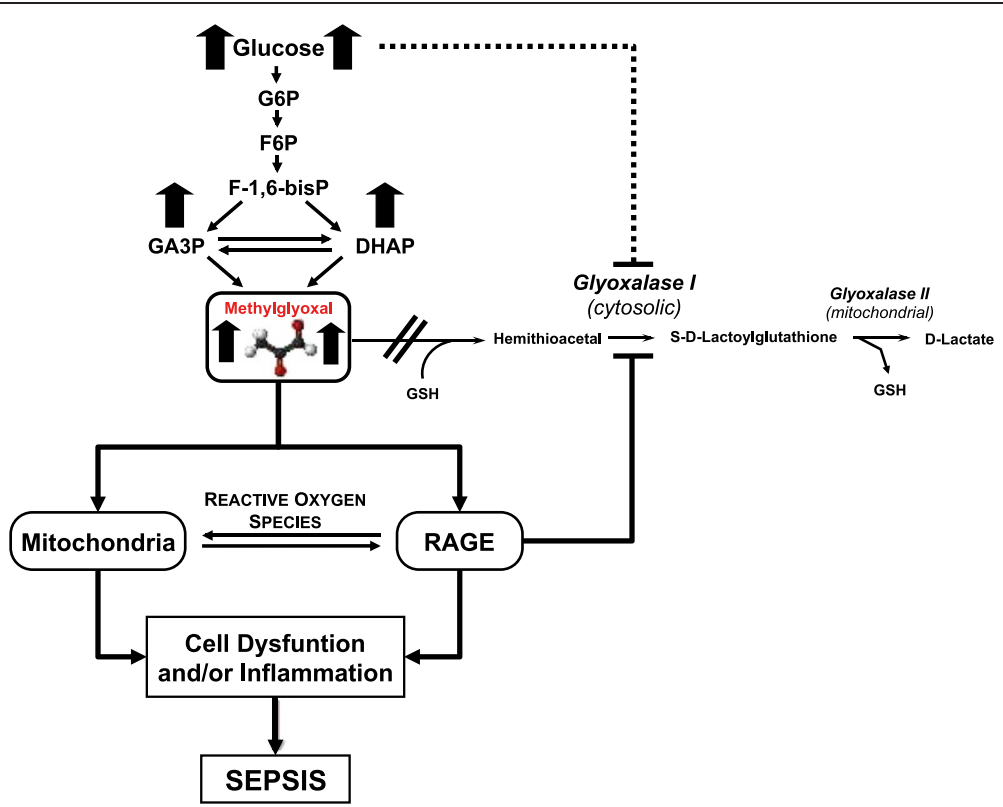

Figure 5 Mechanism of methylglyoxal and methylglyoxal-derived advanced glycation end products driven cell dysfunction or inflammation or both in human sepsis. DHAP, dihydroxyacetone phosphate; F-1,6-bisP, fructose-1,6-bisphosphate; F6P, fructose 6-phosphate; G6P, glucose 6-phosphate; GA3P, glyceraldehyde 3-phosphate; GSH, glutathione; RAGE, receptor for advanced glycation end products.

particularly with respect to the proteins or amino acids (or both) modified by MG [26,40].

\section{Conclusions}

The role of RCS, such as MG, has generally been overlooked in the context of the sepsis syndrome. In this study, MG was identified as a better marker for the identification of patients with sepsis in comparison with routine diagnostic markers. Furthermore, MG was shown to be an early predictor for survival in patients with septic shock. It is hypothesized that in sepsis, increased glucose metabolism resulting from acute hyperglycemia leads to increased production of MG and MG-AGEs. Activation of RAGE by MG-AGEs leads to the downregulation of GLO-1, and a positive feedback loop then leads to further accumulation of MG as well as the transcription of proinflammatory genes. The modification of different (mitochondrial) proteins by MG could lead to increased oxidative stress, further enhancing the MG-activated pathways of cellular dysfunction and inflammation (Figure 5). Further studies are required to determine the extent of MG modification in sepsis and its relative importance to the progression and remission of the condition and whether targeting this pathway would be of therapeutic benefit to the patient.

\section{Key messages}

- MG was identified as a better biomarker than the established routine markers (for example, CRP, PCT,
IL-6, and sCD14-ST) for the identification of patients with sepsis.

- MG was shown to be an early predictor for survival in patients with septic shock.

- The implementation of MG measurements in routine diagnostics of patients with suspected sepsis should therefore be taken into consideration.

- The relative importance of MG to the progression and remission of septicemia, and whether targeting MG-derived carbonyl stress would be of therapeutic benefit to the patient, needs to be further evaluated.

\section{Additional files}

Additional file 1: Table S1. Definitions of infection [13]

Additional file 2: Table S2. Results of pairwise comparisons of plasma levels of C-reactive protein, procalcitonin, interleukin-6, soluble CD14 subtype, blood glucose, and total antioxidant capacity in the postoperative (P/T1, P/T2) and septic (S/TO, S/T1) groups.

Additional file 3: Figure S1. Prediction of short-term survival in patients with septic shock based on methylglyoxal plasma levels. (a) Comparison of plasma methylglyoxal measurements in patients with septic shock who ultimately did and did not survive within the 28-day observation period. Plasma levels of methylglyoxal in surviving and non-surviving patients with septic shock are presented at sepsis onset (S/T0), 24 hours (S/T1), 4 days (S/T2), 7 days (S/T3), 14 days (S/T4), and 28 days (S/T5). Data in bar charts are presented as medians and 95\% confidence intervals (Cls). With regard to symbolism and higher orders of significance: ${ }^{*} P<0.01$. (b) Receiver operating characteristic curve for plasma levels of methylglyoxal (continuous line) at sepsis onset in patients with septic shock who ultimately did and did not survive within the 28 days observation period.

Additional file 4: Figure S2. Prediction of medium-term survival in patients with septic shock based on methylglyoxal plasma levels. 
(a) Comparison of plasma methylglyoxal measurements in patients with septic shock who ultimately did and did not survive within the 90-day observation period. Plasma levels of methylglyoxal in surviving and non-surviving patients with septic shock are presented at sepsis onset (S/T0), 24 hours (S/T1), 4 days (S/T2), 7 days $(\mathrm{S} / \mathrm{T} 3), 14$ days $(\mathrm{S} / \mathrm{T} 4)$, and 28 days (S/T5). Data in bar charts are presented as medians and $95 \%$ confidence intervals (Cls). With regard to symbolism and higher orders of significance: ${ }^{*} P<0.05$. (b) Receiver operating characteristic curve for plasma levels of methylglyoxal (continuous line) at sepsis onset in patients with septic shock who ultimately did and did not survive within the 90-day observation period.

\section{Abbreviations}

AGE: advanced glycation end product; AUC: area under the curve; CRP: C-reactive protein; Ct: cycle threshold; ELISA: enzyme-linked immunosorbent assay; GLO-1: glyoxalase-l; GSH: glutathione; ICU: intensive care unit; L: interleukin; MG: methylglyoxal; MG-AGE: methylglyoxal-derived advanced glycation end products; MG-H1: methylglyoxal-derived hydroimidazolone; PBMC: peripheral blood mononuclear cell; PCR: polymerase chain reaction; PCT: procalcitonin; RAGE: receptor for advanced glycation end products; RCS: reactive carbonyl species; ROC: receiver operating characteristic; ROS: reactive oxygen species; sCD14-ST: soluble CD14 subtype; TAC: total antioxidant capacity.

\section{Competing interests}

The authors declare that they have no competing interests and that they have full control of all primary data. The authors agree to allow the journal to review their data if requested.

\section{Authors' contributions}

TBre was involved in the conception, hypothesis delineation, and design of the study. He performed data acquisition and data analysis and wrote the manuscript. TF was involved in the conception, hypothesis delineation as well as design of the study and helped to write the manuscript. FU, SS, FS, and ES performed data acquisition and were involved in revising the manuscript critically. AU and SZ participated in the design of the study and revised the manuscript critically. TBru participated in the design of the study and performed the statistical analyses. EM, PPN, MAW, and SH conceived the study, participated in its design, and coordinated and helped to draft the manuscript. AB conceived of the study and participated in its design. All authors read and approved the final manuscript.

\section{Authors' information}

SH and MAW share senior authorship.

\section{Acknowledgments}

The authors gratefully acknowledge Ute Krauser, Roland Galmbacher, Serap Kaymak, Anja Buhl, and Divija Deshpande for their excellent technical assistance. This investigation was carried out with financial resources of the Department of Anesthesiology (University of Heidelberg, Germany), the Department of Medicine I and Clinical Chemistry (University of Heidelberg), the Department of Surgery (University of Heidelberg), the Department of Infectious Diseases (University of Heidelberg), and the Institute of Medical Biometry and Informatics (University of Heidelberg).

\footnotetext{
Author details

'Department of Anesthesiology, University of Heidelberg, 110, Im Neuenheimer Feld, D-69120 Heidelberg, Germany. ${ }^{2}$ Department of Medicine I and Clinical Chemistry, University of Heidelberg, 410, Im Neuenheimer Feld, D-69120 Heidelberg, Germany. ${ }^{3}$ Department of General and Transplant Surgery, University of Heidelberg, 110, Im Neuenheimer Feld, D-69120 Heidelberg, Germany. ${ }^{4}$ Department of Infectious Diseases, University of Heidelberg, 324, Im Neuenheimer Feld, D-69120 Heidelberg, Germany. ${ }^{5}$ Institute of Medical Biometry and Informatics, University of Heidelberg, 305, Im Neuenheimer Feld, D-69120 Heidelberg, Germany.
}

Received: 21 May 2014 Accepted: 20 November 2014

Published online: 12 December 2014

\section{References}

1. Angus DC, Linde-Zwirble WT, Lidicker J, Clermont G, Carcillo J, Pinsky MR: Epidemiology of severe sepsis in the United States: analysis of incidence, outcome, and associated costs of care. Crit Care Med 2001, 29:1303-1310.

2. Annane D, Aegerter P, Jars-Guincestre MC, Guidet B: Current epidemiology of septic shock: the CUB-Rea Network. Am J Respir Crit Care Med 2003, 168:165-172.

3. Galley HF: Oxidative stress and mitochondrial dysfunction in sepsis. $\mathrm{Br} J$ Anaesth 2011, 107:57-64.

4. Rocha M, Herance R, Rovira S, Hernandez-Mijares A, Victor VM: Mitochondrial dysfunction and antioxidant therapy in sepsis. Infect Disord Drug Targets 2012, 12:161-178.

5. O'Brien PJ, Siraki AG, Shangari N: Aldehyde sources, metabolism, molecular toxicity mechanisms, and possible effects on human health. Crit Rev Toxicol 2005, 35:609-662.

6. Rabbani N, Thornalley PJ: Methylglyoxal, glyoxalase 1 and the dicarbonyl proteome. Amino Acids 2012, 42:1133-1142.

7. Phillips SA, Thornalley PJ: The formation of methylglyoxal from triose phosphates. Investigation using a specific assay for methylglyoxal. Eur $J$ Biochem 1993, 212:101-105.

8. Thornalley PJ: Modification of the glyoxalase system in human red blood cells by glucose in vitro. Biochem J 1988, 254:751-755.

9. Beisswenger PJ, Howell SK, Nelson RG, Mauer M, Szwergold BS: Alphaoxoaldehyde metabolism and diabetic complications. Biochem Soc Trans 2003, 31:1358-1363.

10. Van Cromphaut SJ, Vanhorebeek I, Van den Berghe G: Glucose metabolism and insulin resistance in sepsis. Curr Pharm Des 2008, 14:1887-1899.

11. Marik PE, Raghavan M: Stress-hyperglycemia, insulin and immunomodulation in sepsis. Intensive Care Med 2004, 30:748-756.

12. Levy MM, Fink MP, Marshall JC, Abraham E, Angus D, Cook D, Cohen J, Opal SM, Vincent JL, Ramsay G: SCCM/ESICM/ACCP/ATS/SIS International Sepsis Definitions Conference. Crit Care Med 2001, 2003:1250-1256.

13. Calandra T, Cohen J: The international sepsis forum consensus conference on definitions of infection in the intensive care unit. Crit Care Med 2005, 33:1538-1548.

14. McLellan AC, Phillips SA, Thornalley PJ: The assay of methylglyoxal in biological systems by derivatization with 1,2-diamino-4,5dimethoxybenzene. Anal Biochem 1992, 206:17-23.

15. Thornalley PJ, Langborg A, Minhas HS: Formation of glyoxal, methylglyoxal and 3-deoxyglucosone in the glycation of proteins by glucose. Biochem $J$ 1999, 344:109-116.

16. DeLong ER, DeLong DM, Clarke-Pearson DL: Comparing the areas under two or more correlated receiver operating characteristic curves: a nonparametric approach. Biometrics 1988, 44:837-845.

17. Pierrakos C, Vincent JL: Sepsis biomarkers: a review. Crit Care 2010, 14:R15.

18. Thornalley PJ: Glyoxalase I-structure, function and a critical role in the enzymatic defence against glycation. Biochem Soc Trans 2003, 31:1343-1348.

19. Abordo EA, Minhas HS, Thornalley PJ: Accumulation of alpha-oxoaldehydes during oxidative stress: a role in cytotoxicity. Biochem Pharmacol 1999, 58:641-648.

20. Bierhaus A, Stoyanov S, Haag GM, Konrade I: RAGE-deficiency reduced diabetes-associated impairment of glyoxalase-1 in neuronal cells. Diabetes 2006, 55:A511

21. Rabbani N, Thornalley PJ: Glyoxalase in diabetes, obesity and related disorders. Semin Cell Dev Biol 2011, 22:309-317.

22. Ahmed N, Dobler D, Dean M, Thornalley PJ: Peptide mapping identifies hotspot site of modification in human serum albumin by methylglyoxal involved in ligand binding and esterase activity. J Biol Chem 2005, 280:5724-5732.

23. Dobler D, Ahmed N, Song L, Eboigbodin KE, Thornalley PJ: Increased dicarbonyl metabolism in endothelial cells in hyperglycemia induces anoikis and impairs angiogenesis by RGD and GFOGER motif modification. Diabetes 2006, 55:1961-1969.

24. Xue J, Ray R, Singer D, Bohme D, Burz DS, Rai V, Hoffmann R, Shekhtman A: The receptor for advanced glycation end products (RAGE) specifically recognizes methylglyoxal-derived AGEs. Biochemistry 2014, 53:3327-3335

25. Karachalias N, Babaei-Jadidi R, Rabbani N, Thornalley PJ: Increased protein damage in renal glomeruli, retina, nerve, plasma and urine and its prevention by thiamine and benfotiamine therapy in a rat model of diabetes. Diabetologia 2010, 53:1506-1516. 
26. Thornalley PJ, Battah S, Ahmed N, Karachalias N, Agalou S, Babaei-Jadidi R, Dawnay A: Quantitative screening of advanced glycation endproducts in cellular and extracellular proteins by tandem mass spectrometry. Biochem J 2003, 375:581-592.

27. Vander Jagt DL: Methylglyoxal, diabetes mellitus and diabetic complications. Drug Metabol Drug Interact 2008, 23:93-124.

28. Berner AK, Brouwers O, Pringle R, Klaassen I, Colhoun L, McVicar C, Brockbank S, Curry JW, Miyata T, Brownlee M, Schlingemann RO, Schalkwijk $C$, Stitt AW: Protection against methylglyoxal-derived AGEs by regulation of glyoxalase 1 prevents retinal neuroglial and vasodegenerative pathology. Diabetologia 2012, 55:845-854.

29. Dhar A, Dhar I, Desai KM, Wu L: Methylglyoxal scavengers attenuate endothelial dysfunction induced by methylglyoxal and high concentrations of glucose. Br J Pharmacol 2010, 161:1843-1856.

30. Bierhaus A, Fleming T, Stoyanov S, Leffler A, Babes A, Neacsu C, Sauer SK, Eberhardt M, Schnolzer M, Lasitschka F, Neuhuber WL, Kichko TI, Konrade I, Elvert R, Mier W, Pirags V, Lukic IK, Morcos M, Dehmer T, Rabbani N, Thornalley PJ, Edelstein D, Nau C, Forbes J, Humpert PM, Schwaninger M, Ziegler D, Stern DM, Cooper ME, Haberkorn U, et al: Methylglyoxal modification of Nav1.8 facilitates nociceptive neuron firing and causes hyperalgesia in diabetic neuropathy. Nat Med 2012, 18:926-933.

31. Abordo EA, Thornalley PJ: Synthesis and secretion of tumour necrosis factor-alpha by human monocytic THP-1 cells and chemotaxis induced by human serum albumin derivatives modified with methylglyoxal and glucose-derived advanced glycation endproducts. Immunol Lett 1997, 58:139-147.

32. Abordo EA, Westwood ME, Thornalley PJ: Synthesis and secretion of macrophage colony stimulating factor by mature human monocytes and human monocytic THP-1 cells induced by human serum albumin derivatives modified with methylglyoxal and glucose-derived advanced glycation endproducts. Immunol Lett 1996, 53:7-13.

33. Webster L, Abordo EA, Thornalley PJ, Limb GA: Induction of TNF alpha and IL-1 beta mRNA in monocytes by methylglyoxal- and advanced glycated endproduct-modified human serum albumin. Biochem Soc Trans 1997, 25:250S.

34. Westwood ME, Argirov OK, Abordo EA, Thornalley PJ: Methylglyoxalmodified arginine residues-a signal for receptor-mediated endocytosis and degradation of proteins by monocytic THP-1 cells. Biochim Biophys Acta 1997, 1356:84-94.

35. Westwood ME, Thornalley PJ: Induction of synthesis and secretion of interleukin 1 beta in the human monocytic THP-1 cells by human serum albumins modified with methylglyoxal and advanced glycation endproducts. Immunol Lett 1996, 50:17-21.

36. Dellinger RP, Levy MM, Rhodes A, Annane D, Gerlach H, Opal SM, Sevransky JE, Sprung CL, Douglas IS, Jaeschke R, Osborn TM, Nunnally ME, Townsend SR, Reinhart K, Kleinpell RM, Angus DC, Deutschman CS, Machado FR, Rubenfeld GD, Webb SA, Beale RJ, Vincent JL, Moreno R: Surviving sepsis campaign: international guidelines for management of severe sepsis and septic shock: 2012. Crit Care Med 2012, 41:580-637.

37. Xue M, Rabbani N, Momiji H, Imbasi P, Anwar MM, Kitteringham N, Park BK Souma T, Moriguchi T, Yamamoto M, Thornalley PJ: Transcriptional control of glyoxalase 1 by Nrf2 provides a stress-responsive defence against dicarbonyl glycation. Biochem J 2012, 443:213-222.

38. Xue M, Qian Q, Adaikalakoteswari A, Rabbani N, Babaei-Jadidi R, Thornalley PJ: Activation of NF-E2-related factor-2 reverses biochemical dysfunction of endothelial cells induced by hyperglycemia linked to vascular disease. Diabetes 2008, 57:2809-2817.

39. Rabbani N, Thornalley PJ: Dicarbonyls linked to damage in the powerhouse: glycation of mitochondrial proteins and oxidative stress. Biochem Soc Trans 2008, 36:1045-1050.

40. Rabbani N, Thornalley PJ: Measurement of methylglyoxal by stable isotopic dilution analysis LC-MS/MS with corroborative prediction in physiological samples. Nat Protoc 2014, 9:1969-1979.

\section{Submit your next manuscript to BioMed Central and take full advantage of:}

- Convenient online submission

- Thorough peer review

- No space constraints or color figure charges

- Immediate publication on acceptance

- Inclusion in PubMed, CAS, Scopus and Google Scholar

- Research which is freely available for redistribution

Submit your manuscript at www.biomedcentral.com/submit 